\title{
Optimised control using Proportional-Integral-Derivative controller tuned using internal model control
}

\author{
B. Mabu Sarif ${ }^{1}$, D. V. Ashok Kumar' ${ }^{2}$ M. Venu Gopala Rao ${ }^{3}$ \\ ${ }^{1}$ Department of Electrical and Electronics Engineering, Koneru Lakshmaiah Education Foundation, India \\ ${ }^{2}$ Rajeev Gandhi Memorial College of Engineering and Technology, India \\ ${ }^{3}$ PVP Siddhartha Institute of Technology, India
}

\begin{tabular}{l} 
Article Info \\
\hline Article history: \\
Received Jun 29, 2019 \\
Revised Nov 11, 2019 \\
Accepted Nov 26, 2019 \\
\hline
\end{tabular}

Keywords:

Disturbance rejection

Filter

IMC

PID

Setpoint tracking

\begin{abstract}
Time delays are generally unavoidable in the designing frameworks for mechanical and electrical systems and so on. In both continuous and discrete schemes, the existence of delay creates undesirable impacts on the underthought which forces exacting constraints on attainable execution. The presence of delay confounds the design structure procedure also. It makes continuous systems boundless dimensional and also extends the readings in discrete systems fundamentally. As the Proportional-IntegralDerivative (PID) controller based on internal model control is essential and strong to address the vulnerabilities and aggravations of the model. But for an real industry process, they are less susceptible to noise than the PID controller.It results in just one tuning parameter which is the time constant of the closed-loop system $\lambda$, the internal model control filter factor. It additionally gives a decent answer for the procedure with huge time delays. The design of the PID controller based on the internal model control, with approximation of time delay using Pade' and Taylor's series is depicted in this paper. The first order filter used in the design provides good set-point tracking along with disturbance rejection.
\end{abstract}

Copyright $@$ C 2020Institute of Advanced Engineering and Science. All rights reserved.

\section{Corresponding Author:}

B. Mabu Sarif,

Department of Electrical and Electronics Engineering,

Koneru Lakshmaiah Education Foundation,

Vaddeswaram, Guntur, Andhra Pradesh, India.

Email: gsn.bms786@gmail.com

\section{INTRODUCTION}

The Controller design is the most indispensable and vital part of the control applications. There are many types of controller architectures which are accessible in the literature. The nature of the controller can be either of conventional or intelligent type. The performance evaluation part comes into action after the design of the controller. The designed controller needs to produce best possibleoutcome inspite of non linearities in plant and equipment and saturation of saturation [1-6].

Kravaris et al. [7] have used Smith predictor as the dead time compensation method for linear systems represented by transfer functions. Gao et al. [8] obtained a PID controller design method based on IMC, it is eye-catching to industrial users because of single alteration parameter. The tuining parameter relate straight to the closed-loop performance and the robustness. Morari-Zafiriou [9] and Gopi et al. [10, 11] have used a first order Pade' approximation of the delay element in the process model in order to realise the closed-loop controller based on IMC principle. This closed-loop controller provides good setpoint response. Horn et al. [12] and Gopi et al. [13] have confirmed the widely published IMC-PID tuning rules provide deprived load disturbance support for applications where the closed-loop dynamics needed are significantly faster than the open loop dynamics. The IMC filter design is tailored to acquire loworder controllers that provide efficient disturbance restraint regardless of where the disturbances are entering 
the closed-loop system.Lee et al. [14] explained PID parameters for general process models by recalling the feedback form in the Laplace variable of an IMC controller with a Maclaurin series. The PID parameters provide closed-loop results nearer to the required outcomes than those acquired by PID controllers tuned by previous methods. A number of PID and predictive controller strategies have been weighed by Syder et al. [15] to compensate for processes modeling in first order lag plus time delay form. The resulting compensated systems 'performance and robustness are evaluated analytically (if applicable) and in simulation. The analytical tuning rules presented by Skogestad et al. [16] they are as easy as possible, resulting in excellent closed-loop behaviour. The guiding principle was the IMC-PID tuning rules of Rivera, Morari and Skogestad that attained wide industrial acceptance. The integral word has been altered to enhance rejection of disturbance in integration systems [17].

Mann et al. [18] described the PID analysis of the time domain, which included three types FOPTD models: (a) negligible time delay, (b) small to medium delay and (c) prolonged delay. The first part of the analysis shows that for plants with negligible time delay, the optimum PID controller is a PI controller. A new PID tuning scheme has been developed for low to medium delay problems. The proposed tuning rule can accommodate the saturation of the actuator and is therefore capable of selecting an optimal PID controller [19]. Chen et al. [20] presented a PID controller design method based on the direct synthesis approach and specification of the desired closed-loop transfer function for disturbance. Analytical expressions for PID controllers are obtained for several popular kinds of process designs including first order, second order plus time delay models and an integer plus time delay model.Skogestad et al. [21] introduced IMC-based tuning guidelines for PID controllers that are easy and still result in excellent loop behavior. To obtain this model form Simple model reduction analytical rules are provided, for effective time delay incorporating the' half rule'. Wang et al. [22] explained recently developed control methods for unstable processes with time delays. The evaluation was carried out using seven existing controller design methods relating to their applicability, control performance and robustness.

Shamsuzzoha et al. [23] proved the IMC-PID tuning laws for excellent set-point monitoring but slow disturbance, which becomes serious when a process has a slight continual delay/time ratio. In this research, an ideal IMC filter structure is suggested for several representative process models to design a PID controller that generates an enhanced reaction to disturbance rejection. A closed-loop guideline is also suggested to cover a broad variety of process models with different time dealy time constant ratio $(\theta / \tau)$.

\section{TUNING RULES FOR FIRSTORDER PROCESS WITH DEAD TIME}

To display the desired property on the control system, controller parameters need to be adjusted. It's known as tuning. In many industries, PID controllers are used. Most of these controllers were analog, but digital signals and computers are used by today's controllers. The controller's parameters can be determined explicitly when a system's mathematical model is available. The parameters are determined experimentally when a mathematical model is not accessible. The parameters of the controller produce the desired controller output. Controller tuning enables a process to be optimised and minimizes the error between the process variable and its set point [1, 6, 24-26].

The different types of methods for controller tuning include methods for testing and error and methods for process reaction curve. Ziegler-Nichols and Cohen-Coon methods are the most common classical controller tuning methods. These techniques are often used when the mathematical model of the system is not accessible. The Ziegler-Nichols technique can be used for both closed and open loop schemes, whereas Cohen-Coon is typically used for open loop schemes. Closed-loop control system is a scheme that utilizes feedback control. The output in an open-loop scheme is not compared to the input $[1,6,11,24,26]$. The equation of the PID controller is represented in (1).

$$
u(t)=K_{P}\left[e(t)+\frac{1}{T_{i}} \int e(t) d t+T_{d} \frac{d}{d t} e(t)\right]
$$

There are three tuning parameters for a PID controller. If these are adjusted in an adhoc fashion, a satisfactory controller performance may take a while. Thus, in 1942 Ziegler and Nichols proposed the two tuning methods [1] and were widely used either in the original form or in modified forms [5]. First method is called the ultimate method of sensitivity by Ziegler-Nichols. The second method is called the step response method of Ziegler-Nichols [1, 5, 6, 24].

The prior tuning rules were based on experiments that compelled a process to continuously oscillate. As a consequence, the system is compelled to the brink of instability and it may take some time to alliteratively adjust the controller for constant oscillation. The tuning rules explained below are based on 
the process models which are represented as FOPDT process of (2) or (3), which are obtained through open loop step tests.

$$
G_{P}(s)=K e^{-\theta s}
$$

The first order plus time delay processes have a maximum slope of $K=K_{P} / \tau_{P}$ at $t=q$ these guidelines can be used in the first order plus time delay processes for a unit step input.

$$
G_{P}(s)=K e^{-\theta s} /\left(\tau_{p} s+1\right)
$$

\section{PROPORTIONAL INTEGRAL DERIVATIVE CONTROLLER TUNING WITH INTERNAL MODEL CONTROL}

A model-based control method is used in Internal Model Control (IMC). It is also possible to use the IMC method as a PIDcontroller tuning method [7, 11 25, 27]. The method is generally applicable to systems with constant delays, but the IMC method is also applied to systems with varying time delays. Photograph Figure 1 is the IMC principle representation.

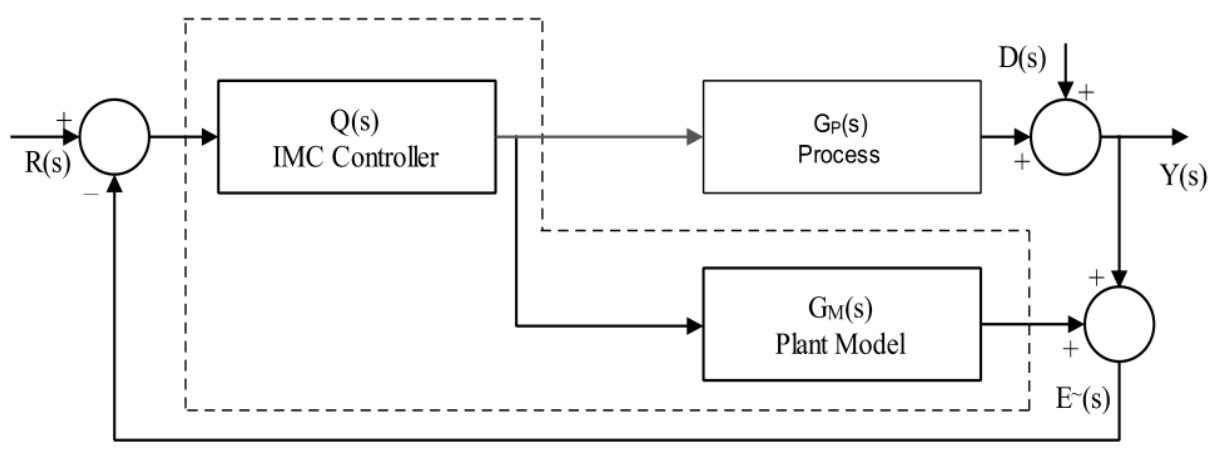

Figure 1. IMC structure [3]

The model output error is removed from the reference signal and fed into the control signal calculating IMC. Calculating the IMC controller $\mathrm{Q}(\mathrm{s})$ first divides the process model into two parts as follows:

$$
\tilde{G}_{M}(s)=\tilde{G}_{M^{+}}(s) \tilde{G}_{M^{-}}(s)
$$

$\tilde{G}_{M^{+}}(s)$ Is the model's non-invertible part, Includes all unstable zeroes and delays. Included in the rest of the model $\tilde{G}_{M^{-}}(s)$. The IMC controller $Q(s)$ is given by (5):

$$
Q(s)=\left[\tilde{G}_{M-}(s)\right]^{-1} f(s)
$$

where $f(s)$ the transfer function of low-pass with order $n(6)$ :

$$
f(s)=\frac{1}{\left(1+\lambda_{I M C} s\right)^{n}}
$$

To have a causal controller, the low-pass filter is required. The $\lambda_{I M C}$ is the IMC method's tuning parameter. The value $\lambda_{I M C}$ of the controlled system has a important impact on efficiency and robustness. 
It is difficult to achieve robust tuning and fast response simultanesouly, thus thieis a trade-off. Robustness plays a vital role in different time-delay systems, and it turns out that tuning $\lambda_{I M C}$ is crucial.

Recognizing the dependence between the IMC controller Q in Figure 1 is useful when implementing the IMC controller with the controller of traditional feedback loop in Figure 2. The (7) gives the IMC law in the classic control loop.

$$
G_{C}(s)=\frac{Q(s)}{1-\tilde{G}_{M}(s) Q(s)}
$$

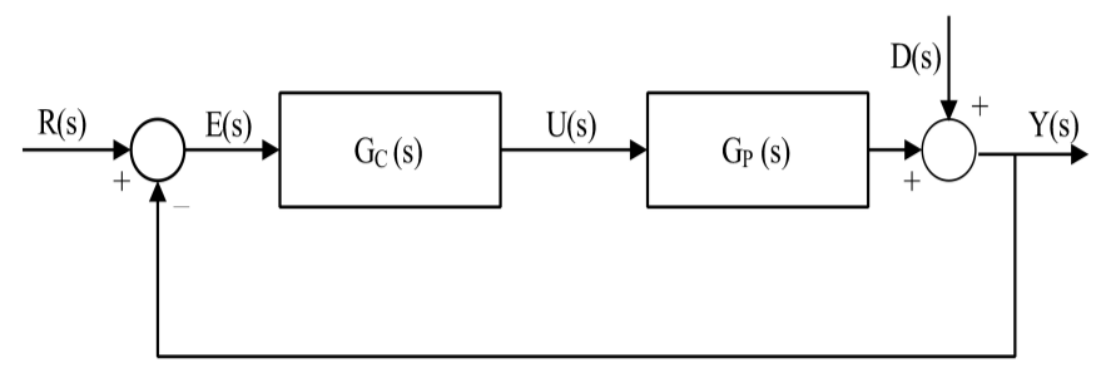

Figure 2. IMC modified structure for closed-loop [3]

The process delays must be approximated with linear transfer function to design the controller. The delay can be approximated to the expansion of the Taylor series or the approximation of the first order Padé [10].

$$
e^{-\theta s}=\frac{(1-\theta s / 2)}{(1+\theta s / 2)}
$$

Under certain assumptions, the IMC design often yields high-order controllers. The proportional integral (PI) control structure can be obtained from the IMC design and the tuning parameters can therefore be obtained for a regular proportional internal control controller. Consider the model for the FOPTD process.

Using the IMC design and the first order Taylor series expansion $e^{-\theta s} \approx(1-\theta s)$ and with first order $(n=1)$ low-pass filter the controller $\mathrm{C}$ becomes $[5,10]$.

$$
C_{P I}(s)=\frac{(1+\tau s)}{K s\left(\lambda_{I M C}+\theta\right)}
$$

The PI controller with parameters is given as:

$$
K_{P}=\frac{\tau}{K\left(\lambda_{I M C}+\theta\right)} ; T_{i}=\tau
$$

When using the delay approximation padé, controller $\mathrm{C}$ becomes [10].

$$
\begin{aligned}
& C_{P I D}(s)=\frac{\tau}{K s\left(\lambda_{I M C}+1\right)}(1+1 / \tau s)(1+\theta s / 2) \\
& K_{P}=\frac{\tau}{K\left(\lambda_{I M C}+\theta\right)}(1+\theta / 2 \tau) ; T_{i}=\tau+\theta / 2 ; T_{d}=\frac{\theta \tau}{2 \tau+\theta}
\end{aligned}
$$

The PI Controller form is in fact the interacting controller $[6,10,11]$. 


\section{THE IMC BASED PID CONTROL DESIGN PROCEDURE}

In the design of the IMC based PID control system, the following steps are used [11, 24]:

- First, it is necessary to determine the transfer function of the IMC controller $Q(s)$, including a low pass filter $f(s)$ for semi-proper or derivative action. The numerator's order is one order larger than the denominator and this is required to find a PID controller equivalent. It's a big difference from the process of IMC. A filter of the form (13) is often used for integration or unstable processes or to achieve better disruption rejection $[11,12]$.

$$
f(\mathrm{~s})=1 /\left(\lambda_{I M C^{s}}+1\right)^{n}
$$

- Using the transformation, the equivalent standard feedback controller is given

$$
G_{C}(s)=Q(s) /\left(1-\tilde{G}_{P}(s) Q(s)\right)
$$

- The (14) must be displayed in PID form and evaluated in $K_{P}, T_{i}, T_{d}$. This process sometimes results in an optimal PID controller cascaded with a filter with a steady filter period $\left(\tau_{\mathrm{f}}\right)$.

$$
C(s)=K_{P}\left[T_{i} T_{d} s^{2}+T_{i} s+1\right]\left[1 /\left(\tau_{f} s+1\right)\right]
$$

- The ideal model situation and instances with closed-loop model mismatch simulations need to be performed. Adjust $\lambda_{\mathrm{IMC}}$ to model error based on a tradeoff amid performance and robustness sensitivity. The initial values for $\lambda_{\mathrm{IMC}}$ are between $1 / 3$ and $1 / 2$ of the dominant time constant [24].

\subsection{First order plus dead time process}

The most common representation of chemical process dynamics is the first-order plus dead time. For a large number of process control loops, the PID equivalent form developed is useful. The following steps are used for first order with dead time processes in the IMC-based PID design. The process is given by:

$$
G_{P}(s)=K \frac{e^{-\theta s}}{(1+\tau s)}
$$

- The approximation of the first order for the dead time is provided by [10]

$$
\begin{aligned}
& e^{-\theta s}=(1-0.5 \theta s) /(1+0.5 \theta s) \\
& G_{M}(s)=\frac{K(1-0.5 \theta s)}{(\tau s+1)(1+0.5 \theta s)}
\end{aligned}
$$

- To avoid the predictive part, factor the non-invertible elements

$$
\begin{aligned}
& G_{M-}(s)=K /[(1+\tau s)(1+0.5 \theta s)] \\
& G_{M_{+}}(s)=(1-0.5 \theta s)
\end{aligned}
$$

- Then the idealized controller is given

$$
\tilde{Q}(s)=(\tau s+1)(0.5 \theta s+1) / \mathrm{K}
$$

- To make the $\mathrm{Q}(\mathrm{s})$ proper, filter $\mathrm{f}(\mathrm{s})$ is added. But to get the PID controller, $\mathrm{Q}(\mathrm{s})$ will besemi-proper. The derivative option is used to allow $\mathrm{Q}(\mathrm{s})$ numerator to be higher than the denominator in one order.

$$
Q(s)=\tilde{Q}(s) f(s)=G_{M-}^{-1}(s) f(s)
$$


The PID equivalent is obtained as:

$$
\begin{aligned}
G_{C}(s) & =\frac{Q(s)}{\left(1-\tilde{G}_{M}(s) Q(s)\right)} \\
G_{C}(s) & =\frac{1}{K} \frac{(\tau s+1)(1+0.5 \theta s)}{(\lambda s+0.5 \theta s) s} \\
G_{C}(s) & =\frac{1}{K} \frac{0.5 \tau \theta s^{2}+(0.5 \theta+\tau) s+1}{(\lambda+0.5 \theta s) s}
\end{aligned}
$$

PID parameters evaluated from the above equation are shown below:

$$
\begin{aligned}
& K_{P}=\frac{(\tau+0.5 \theta)}{K(\lambda+0.5 \theta)} \\
& T_{i}=\tau+0.5 \theta \\
& T_{d}=\frac{\tau \theta}{2 \tau+\theta}
\end{aligned}
$$

When the process is first order plus dead time, the IMC-based PID controller design procedure has resulted in a PID controller. In this development, a Padé approximation for dead time was used which means that the filter constant $(\lambda)$ can not be randomly reduced. The IMC-based PID strategy will therefore have performance limitations that do not occur in the IMC strategy. Because of the model uncertainty owing to the approximation of thePadé, Rivera et al. (1986) [28] suggest that $\lambda>0.8$ be used owing to the uncertainty of the model. Morari and Zafiriou (1989) suggest $\lambda>0.25$ [9] for the PID plus lag system.

\subsection{Integratorplus deadtime process}

For processes in which the time constant is dominant, the step response behavior can be approximated as an integrator plus dead time as the following transfer function characterizes [17, 29-34].

$$
G_{M}(s)=\frac{K e^{-\theta s}}{s}
$$

Approximating it as FOPTD model [17, 29-34]

$$
G_{M}(s)=\frac{\eta K e^{-\theta s}}{\eta s+1}, \text { where } \eta=\text { Large arbitrary constant }
$$

Applying the First Pade' expansion approximate to the delay term $e^{-\theta s}$ results in

$$
\begin{aligned}
& e^{-\theta s}=\left(\frac{1-0.5 \theta s}{1+0.5 \theta s}\right) \\
& G_{M}^{-}(s)=\frac{\eta K}{(\eta s+1)(1+0.5 \theta s)}, G_{M}^{+}(s)=(1-0.5 \theta s)
\end{aligned}
$$

IMC controller $Q(s)$ is:

$$
Q(s)=\frac{(\eta s+1)(1+0.5 \theta s)}{\eta K} \frac{1}{(\lambda s+1)}
$$


The closed-loop feedback controller, equal to IMC controller $Q(s)$ is:

$$
\begin{aligned}
G_{C}(s) & =\frac{Q(s)}{1-Q(s) G_{M}(s)} \\
G_{C}(s) & =\frac{(\eta s+1)(1+0.5 \theta s)}{\eta K[(\lambda s+1)(1+0.5 \theta s)-(1-0.5 \theta s)]}
\end{aligned}
$$

A proportional integral controller results with the following parameters for integral process.

$$
K_{P}=\frac{(\eta+0.5 \theta)}{\eta K(\lambda+0.5 \theta)} ; T_{i}=\eta+0.5 \theta ; T_{d}=\frac{0.5 \theta \eta}{\eta+0.5 \theta}
$$

\section{RESULTS AND ANALYSIS}

Design with time delay is implemented using MATLAB in the IMC-based PID controller. The actual function of process transfer is never accurately known. It is therefore necessary to use two process representations of the transfer function. Thus, one is regarded as a process or plant that is never accurately known, and the other is considered as a process model that is accurately known. In the IMC process model, the actual process is maintained in parallel. The ideal PID controller based on IMC means that the model is perfect and there is no disturbance or delay. So there's no feedback either.

\subsection{Case 1: FOPDT}

An IMC based PID controller's transfer function is given in (37) for a first order with time delay plus first order disturbance. The function of transfer is taken from [25]. The approximation of a first order Pade' is used for time delay. A first-order disturbance $G_{d}(s)$ (38) together with the process model is considered.

$$
\begin{aligned}
& G_{P}(s)=\frac{100 e^{-1 s}}{(100 s+1)} \\
& G_{d}(s)=\frac{1}{(30 s+1)}
\end{aligned}
$$

The time delay is approximated with first order Pade' approximation and the value of IMC filter $f(s)=1 /(1+\lambda s)^{2}$ to make the controller semi-proper. The value of $\lambda=20$ is chosen, which is having range $\lambda>0.2 \tau$. But practically the initial values of $\lambda$ should lie in the range of $1 / 3$ to $1 / 5$ of the time constant of the process. Substituting the value of $\lambda$ in the IMC controller $Q(s)$ of (21) along with (36), (37), (17), (18) and (19) results in (39).

$$
Q(s)=\frac{\left(50 s^{2}+100.5 s+1\right)}{\left(400 s^{2}+40 s+1\right)}
$$

For obtaining the closed-loop feedback controller with PID controller, substitution and simplification with the procedure defined earlier the parameters of the PID controller are :

$$
K_{P}=0.049 ; T_{i}=100.5 ; T_{d}=0.5
$$

IMC-based PID controller's Simulink block diagram for a first order with time delay plus first order disruption is shown in Figures 3 and 4. The IMC based PID controller's unit step response is shown in Figure 5 for a first order with time delay plus first order disturbance. Photograph. Figures 6 and 7 illustrate the disturbance response of IMC-PID and IMC, and Table 1 encapsulates the integral performance criteria for FOPTD plus disturbance of the first order. From Figures 5, 6, 7 and Table 1 it can be inferred, in contrast to the IMC controller, the IMC-PID provides improved set point tracking and disturbance rejection. Rising time is improved, settling time is reduced, and disturbance recovery is rapid. 


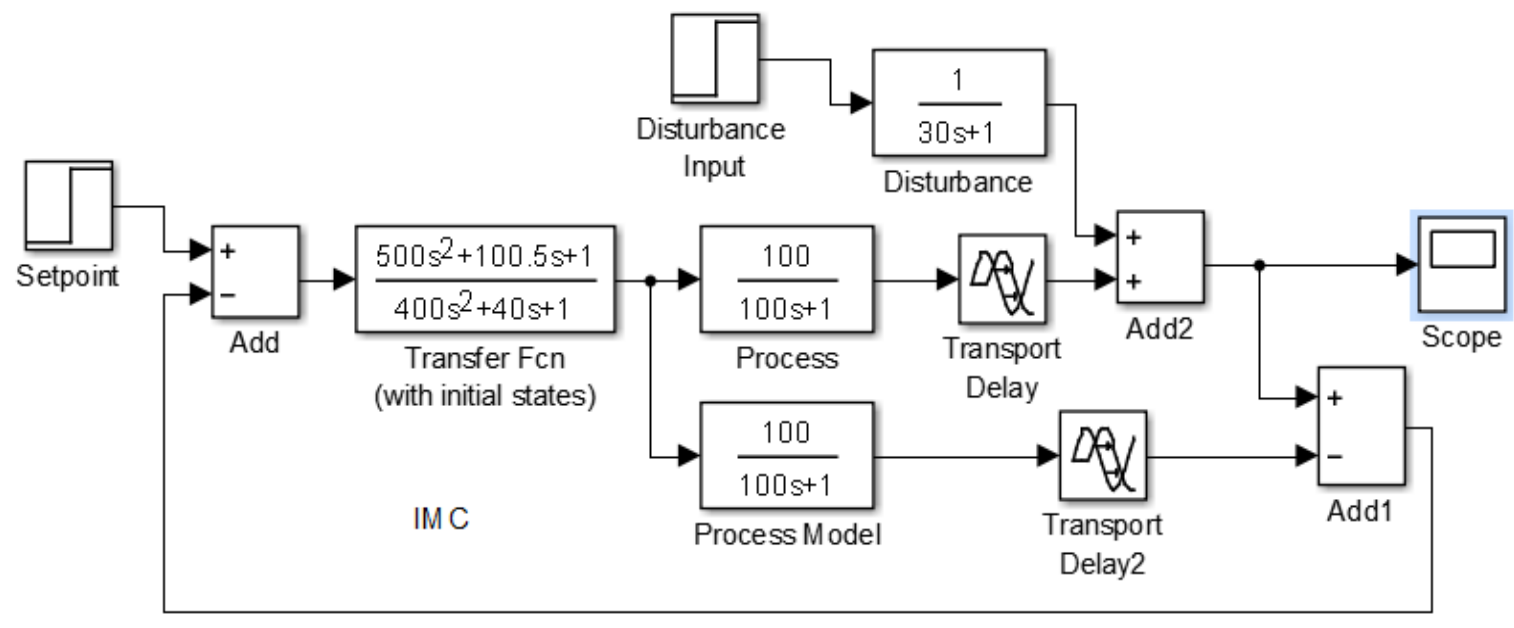

Figure 3. Simulink implementation of IMC for FOPTD

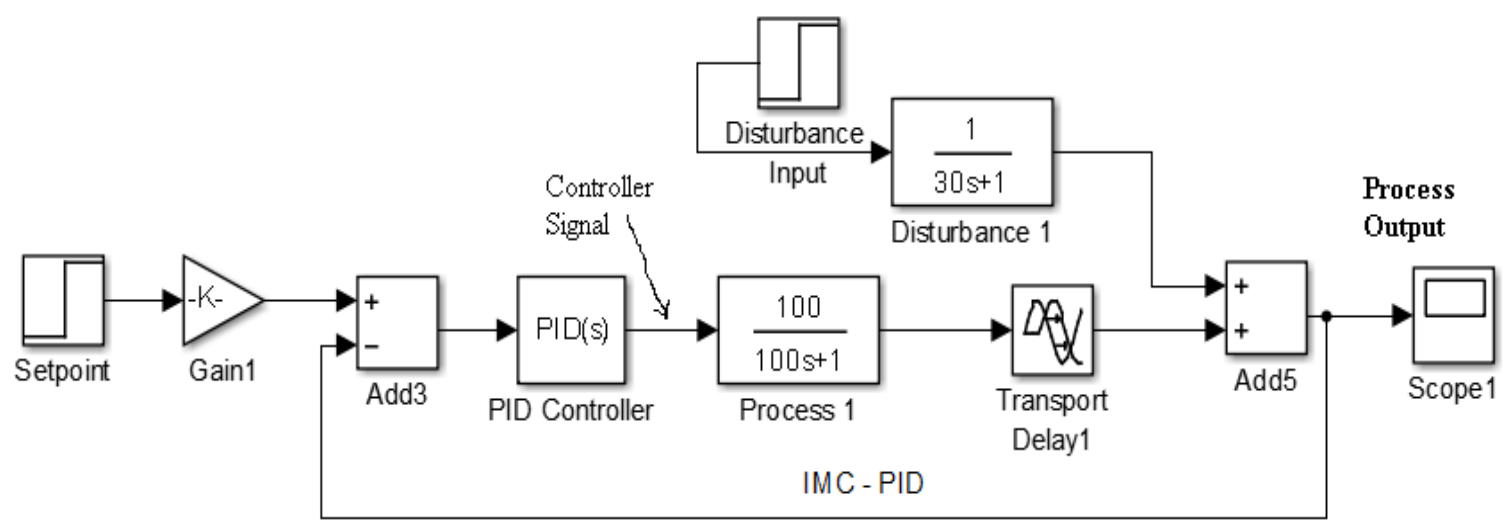

Figure 4. Simulink implementation of IMC-PID for FOPTD

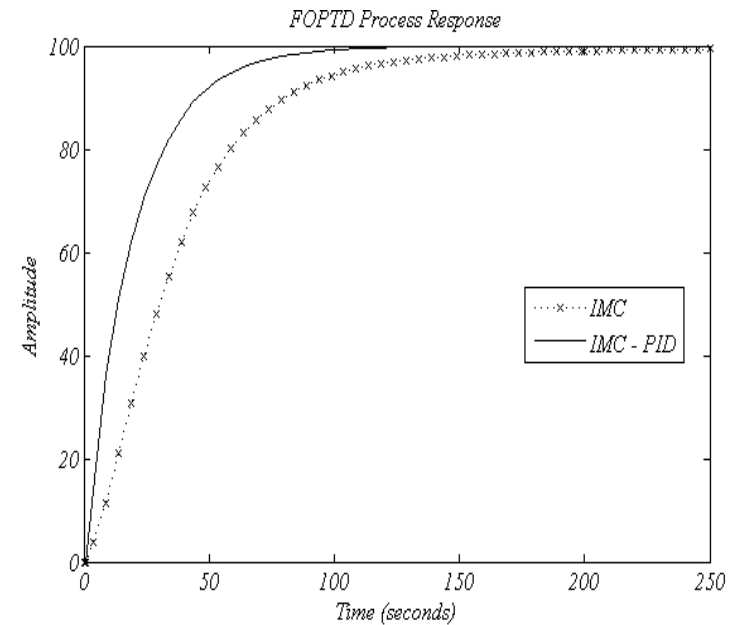

Figure 5. Setpoint Response of FOPTD for step input

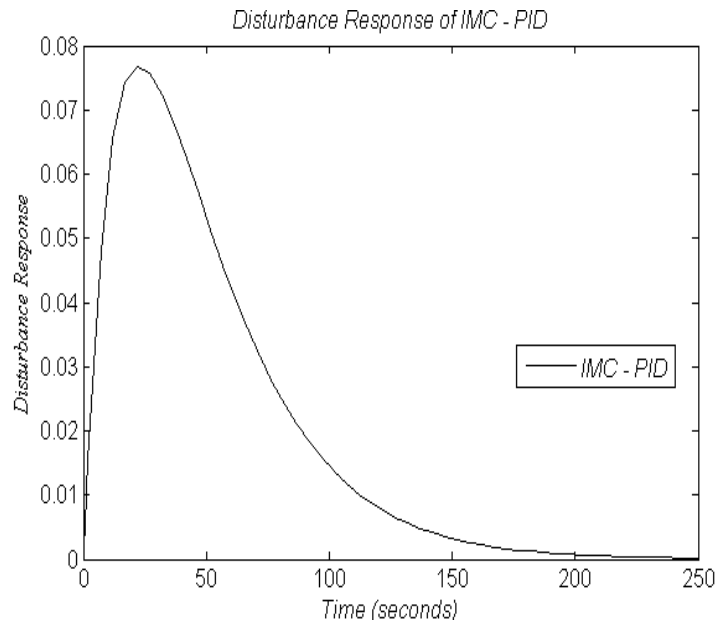

Figure 6. Disturbance response of IMC-PID for FOPTD 


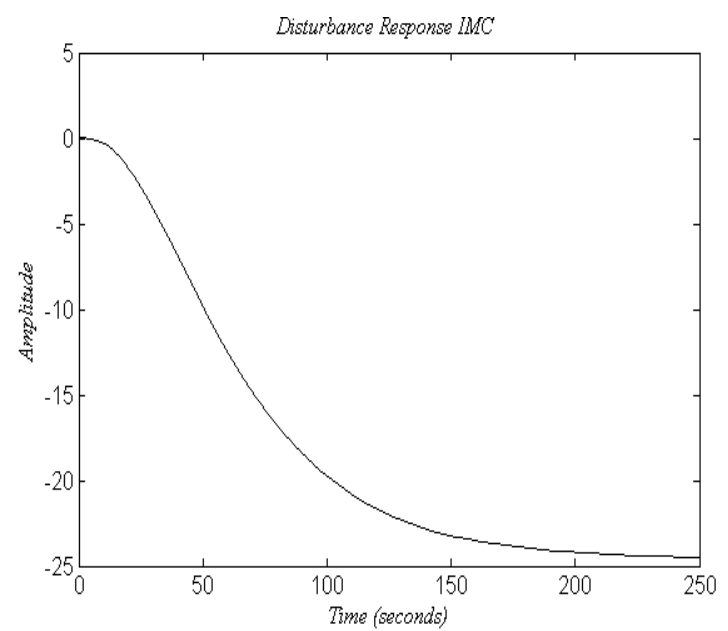

Figure 7. Disturbance response of IMC for FOPTD

Table 1. Performance comparison of FOPTD

\begin{tabular}{lcccccc}
\hline & \multicolumn{3}{c}{ Disturbance } & \multicolumn{3}{c}{ Setpoint } \\
\hline Controller & IAE & ISE & ITAE & IAE & ISE & ITAE \\
IMC & 55 & 12.81 & 758.8 & 249 & 249 & 3125 \\
IMC-PI/PID (Proposed) & 5.122 & 0.266 & 255.5 & 20.51 & 10.54 & 439.7 \\
\hline
\end{tabular}

\subsection{Case 2: Integrating process}

The process model of integrating process with time delay is considered [17],

$$
G_{M}(s)=\frac{0.2 e^{-7.4 s}}{s}
$$

For obtaining the closed-loop feedback controller with PID controller, substitution and simplification with the procedure defined earlier in the section IV, the parameters of the PID controller are:

$$
K_{P}=0.049 ; T_{i}=100.5 ; T_{d}=0.5
$$

The disturbance response of the IMC-PID controller for integrating process is represented in Figure 8. The IMC-PID provides less overshoot and fast recovery from disturbance along with good set point tracking. Figure 9 is the representation of the controller response for disturbance and it is a smooth action thus enhancing the life of process equipment. Table 2 encapsulates the integral performance criterion which demonstrates the performance of the designed IMC-PI/PID controller.

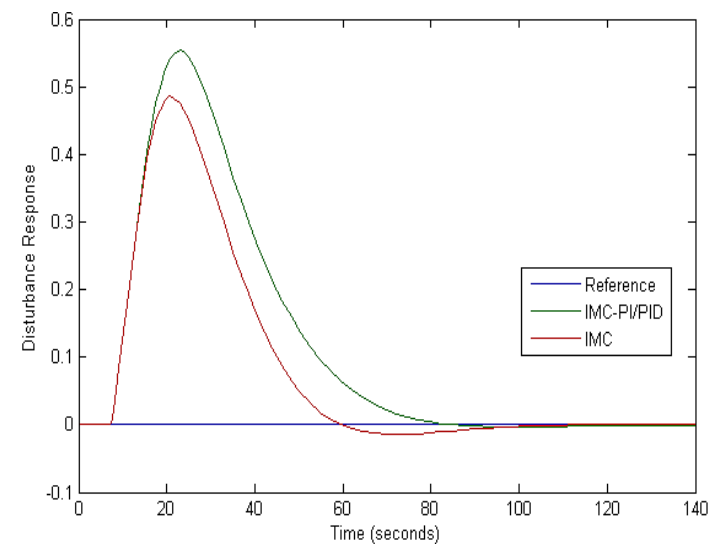

Figure 8. Disturbance response of IMC-PID for integrating process

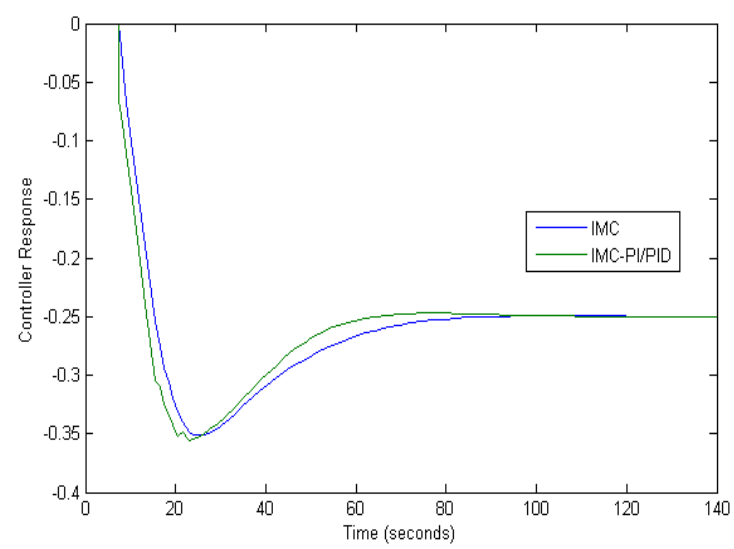

Figure 9. Controller behaviour for integrating process 
Table 2. Performance comparison of integrating process

\begin{tabular}{lcccccc}
\hline & \multicolumn{3}{c}{ Disturbance } & \multicolumn{3}{c}{ Setpoint } \\
\hline Controller & IAE & ISE & ITAE & IAE & ISE & ITAE \\
IMC & 16.34 & 6.158 & 505.4 & 26.95 & 15.66 & 679.4 \\
IMC-PI/PID & 12.29 & 4.123 & 342.8 & 24.01 & 15.49 & 493.2 \\
(Proposed) & 12.2 & & & & &
\end{tabular}

\section{CONCLUSION}

The IMC provides a transparent framework for the design and tuning of control systems. The design of the IMC-based PID controller is simple and robust to handle model uncertainties and disturbances and less noise-sensitive than conventional PID controller for an actual industry process. The design of the IMC based PID controllers results in only one tuning parameter that is the closed-loop time constant $\lambda$ which is the factor of the IMC filter. The parameters of the IMC based PID tuning are then a function of the time constant of the closed-loop. Closed-loop time constant selection is directly related to the closed-loop system's robustness sensitivity to model error. The PID design procedure based on IMC can be implemented using existing PID control equipment in industrial processes. It also provides a good process solution with significant time delays, which is actually the case with real-time work.

\section{REFERENCES}

[1] J. G. Ziegler, N. B. Nichols, "Optimum settings for automatic controllers," Transactions of the ASME, pp. 759-768, 1942.

[2] G. H. Cohen, G. A. Coon, "Theoretical Consideration of Retarded Control," Transactions of the ASME, vol. 65, pp. 827-834, 1953.

[3] C. E. Garcia, M. Morari, "Internal Model Control. A Unifying Review and Some New Results, Industrial \& Engineering Chemistry Process Design and Development," vol. 21, pp. 308-323, 1982.

[4] John Doyle, Bruce Francis, Allen Tannenbaum, "Feedback Control Theory," Macmillan Publishing Co, pp. 31-34, 1990.

[5] K. J. Aström, and T. Hagglund, "PID Controllers: Theory, Design, and Tuning," $2^{\text {nd }}$ ed, Instrument Society of America, 1995.

[6] A. O'Dwyer, Handbook of PI and PID Controller Tuning Rules, Imperial College Press, London, pp. 37, 2003.

[7] Costas Kravaris, Raymond A. Wright, "Deadtime Compensation for Nonlinear Processes," AIChE Journal, vol. 35, no. 9, pp. 1535-1542, 1989.

[8] Gao Jinchang, Zhou Chunhui, Gong Xiaofeng, "Extension of IMC tuning to improve controller performance, Systems, Man, and Cybernetics," IEEE International Conference, vol. 3, pp. 1770-1775, 1996.

[9] Manfred Morari and Evanghelos Zafiriou, Robust Process Control, Prentice Hall, 1989.

[10] Gopi Krishna Rao, P. V., Subramanyam, M. V., and Satyaprasad, K., "Time delay approximation: Its influence on the structure and performance of IMC-PI/PID controller. Intelligent Computing, Communication and Devices, Advances in Intelligent Systems and Computing," Springer India, vol. 308, pp. 561-569, 2015.

[11] Gopi Krishna Rao, P. V., Subramanyam, M. V., and Satyaprasad, K., "Model based tuning of PID controller," Journal of Control \& Instrumentation, vol. 4, no. 1, pp. 16-22, 2013.

[12] Ian G. Horn, Jeffery R. Arulandu, Christopher J. Gombas, Jeremy G. VanAntwerp, and Richard D. Braatz, "Improved filter design in internal model control," Industrial Engineering Chemical Resources, vol. 35, no. 10, pp. 3437-3441, 1996.

[13] Gopi Krishna Rao, P. V., Subramanyam, M. V., and Satyaprasad, K., "Design of cascaded IMC-PID controller with improved filter for disturbance rejection,"International Journal of Applied Science and Engineering, vol. 12, no. 2, pp. 127-141, 2014.

[14] Lee, Y., Park, S., Lee, M., Brosilow, C., "PID controller tuning for desired closed-loop responses for SI/SO systems," AIChE J., vol. 44, no. 1, pp. 106-115, 1998.

[15] Syder J, T Heeg, and A O'Dwyer, "Dead-time Compensators: Performance and Robustness Issues," European Journal of Control, pp. 166-171, 2000.

[16] Sigurd Skogestad, "Probably the best simple PID tuning rules in the world," Journal of Process Control, pp. 1-27, 2001.

[17] P. V. Gopi Krishna Rao, M. V. Subramanyam, K. Satyaprasad, "Robust Design of PID Controller Using IMC Technique for Integrating Process Based on Maximum Sensitivity," Journal of Control Automation and Electrical Systems, SPRINGER, vol. 26, no. 05, pp. 466-475, 2015.

[18] G. K .I. Mann, B.-G.Hu and R. G. Gosine, "Time-domain based design and analysis of new PID tuning rules," IEE Proceedings.-Control Theory Application, vol. 148, no. 3, pp. 251-261, 2001.

[19] Tony Hong, Peter C Hughes, "Effect of Time Delay on the Stability of Flexible Structures with Rate Feedback Control," journal of vibration and control, vol. 7, no. 1, pp. 33-49, 2001

[20] Chen, D., Seborg, D. E., "PI/PID controller design based on direct synthesis and disturbance rejection," Industrial Engineering Chemical Resources, vol. 41, no. 19, pp. 4807-4822, 2002.

[21] Sigurd Skogestad, "Simple Analytic Rules for Model Reduction and PID Cotroller tuning," Journal of Process Control, vol. 13, no. 4, pp. 291-309, 2003. 
[22] Qing-Guo Wang, Han-Qin Zhou, Yu Zhangt and Yong Zhang, "A Comparative Study on Control of Unstable Processes with Time Delay," $5^{\text {th }}$ Asian Control Conference, pp. 1996-2004, 2004.

[23] B Wayne Bequette, Process Control: Modelling, Design, and Simulation, Prentice Hall, pp. 198-206, 294-297, 262-264, 2003

[24] Gopi Krishna Rao, P. V., Subramanyam, M. V., and Satyaprasad, K., "Design of IMC-PID controller with improved filter for disturbance rejection," Systems Science \& Control Engineering: An Open Access Journal, vol. 2, no. 1, pp. 583-592, 2014.

[25] K. J. Aström, and T. Hagglund, "Revisiting the Ziegler-Nichols step response method for PID control," Journal of Process Control, vol. 14, pp. 635-650, 2004.

[26] G. Madhu Kumar, V. Suma Deepthi, "Design and Development of IMC Tuned PID Controller for Disturbance Rejection of Pure Integrating Process," International Journal of Innovative Technologies, vol. 04, no. 06, pp. 1117-1121, Jun. 2016.

[27] Rivera DE, Morari M, Skogestad S., "Internal model control. 4. PID controller design," Ind Eng Chem Process DesDev., vol. 25, pp. 252-265, 1986.

[28] Anusha, A. V. N. L., and Rao, A. S., "Design and analysis of IMC based PID controller for unstable systems for enhanced closed loop performance," Proceedings of the IFAC Conference Advances in PID control (PID"12), vol. 45, no. 3, pp. 41-46, 2012.

[29] Arbogast, J. E., and Cooper, D. J., "Extension of IMC tuning correlations for non-self regulating (integrating) processes," ISA Transactions, vol. 46, no. 3, pp. 303-311, 2007.

[30] Chia, T. L., and Lefkowitz, I., "Internal model-based control for integrating processes," ISA Transactions, vol. 49, no. 4, pp. 519-527, 2010.

[31] Chien, I. L., and Fruehauf, P. S. "Consider IMC tuning to improve performance," Chemical Engineering Progress, vol. 86, pp. 33-41, 1990.

[32] Eris, O., and Kurtulan, S., "A new PI tuning rule for first order plus dead-time systems," IEEE Africon, vol. 11, pp. 1-4, 2011

[33] Panyam Vuppu, G. K. R., Makam Venkata, S., Kodati, S., "Robust Design of PID Controller Using IMC Technique for Integrating Process Based on Maximum Sensitivity," Journal of Control, Automation and Electrical Systems, vol. 26, no. 5, pp. 466-475, 2015.

[34] Hanuma Naik, R., Kumar, D. V. A., Gopikrishna Rao, P. V., "Improved centralised control system for rejection of loop interaction in coupled tank system," Indian Chemical Engineer, vol. 1-20, 2019. 